schwindigkeitszunahme wälı'end der Umdrehung beurtheilen könne. Alle Tafeln sind Umkehrungen der Originale und gehen daher von rechts nach links.

Ausdrücklich bemerke ich, dass die eigentlichen direct auf Papier hergestellten Phonogramme ungleich eleganter und klarer sind, als die a uf Umwegen hergestellten der Tafel. Ich bin gern bereit, Instituten und Fachgenossen auf Verlangen Original-Papierphonogramme zur Verfügung zu stellen.

Der Cylinder wurde in den Versuchen für die Tafel stets mit der Hand gedreht, so dass die Geschwindigkeiten der einzelnen Curven, auch auf derselben Tafel, stets verschieden sind. Da jedoch die Tonhöhe angegeben ist, kann der Lieser jede Geschwindigkeit selbst ermitteln.

Die Bedeutung der Curven ist aus der Schriftbezeichnung genau ersichtlich; auf die wesentlichen Erscheinungen ist im Texte Bezug genommen. Alle Curven, soweit nichts vermerkt ist, rühren von meiner eigenen Stimme her; nur auf Blatt 1,5 und 6 sind die 6 untersten Curven von der Tenorstimme des Herrn Dr. Ehrenthal geliefert. Alle Noten gehören der $G$-dur-Tonleiter an (vgl. oben S. 350).

\title{
Weitere Untersuchungen über den Heliotropismus der Thiere und seine Uebereinstimmung mit dem Heliotropismus der Pflanzen.
}

(Heliotropische Krì mmungen bei Thieren.)

Von

\author{
Dr. J. Loeb,
}

Assistent am physiologischen Institut zu Strassburg i. E.

Hierzu Tafel IX und 2 Holzschnitte.

In einer früheren Arbeit habe ich nachgewiesen, dass die Abhängigkeit thierischer Bewegungen vom Licht die gleiche ist, wie die Abhängigkeit pflanżlicher Bewegungen von derselben Reizursache $^{1}$ ). Ich zeigte, dass das von Sachs für den Heliotropis-

1) Der Heliotropismus der Thiere und seine Uebereinstimmung mit dem Heliotropismus der Pflanzen. Würzburg, 1890. Verlag von Georg Hertz.

Vgl. ferner: Loeb, Die Orientirung der Thiere gegen das Licht (Thie- 
mus der Pflanzen aufgestellte Gesetz, nämlich, dass die Richtung des Lichtstrahls die Orientirung bestimmt, auch für die Thiere gültig ist. Die freibeweglichen Thiere sind gezwungen, ihre Progressiv-Bewegungen in der Richtung des Lichtstrahls auszuführen, wie die Schwärmsporen gewisser Algen. Es liess sich ferner zeigen, dass die stärker brechbaren Strahlen des uns sichtbaren Sonnenspectrums die ausschliesslich, oder doch die vorwiegend bei der Bewegung der Thiere wirksamen sind, wie bei heliotro* pischen Bewegungen der Pflanzen a. s. f. Nachdem ich die Identität des Abhängigkeitsverhältnisses Punkt für Punkt dargethan hatte, hielt ich es für erlaubt, dasselbe bei den Thieren mit demselben Ausdruck zu bezeichnen, mit dem es auch bei den Pflanzen bezeichnet wird, nämlich als Heliotropismus.

Allein der Nachweis der Identität hatte sich damals nur auf freibewegliche Thiere erstreckt. Es blieb Aufgabe weiterer Untersuchungen, den Einfluss des Lichtes auf die Orientirung festsitzender Thiere zu ermitteln und zu priffen, ob der Einfluss des Lichtes hier wiederum der gleiche ist, wie bei festsitzenden Pflanzen. Bei letzteren handelt es sich bekanntlich ebenfalls darum, dass die Richtung des Lichtstrahls die Orientirung des pflanzlichen Organs bestimmt. Charakteristisch für den Heliotropismus festsitzender pflanzlicher Organe ist der Umstand, dass hier bei einseitiger Beleuchtnng heliotropische K rümmungen eintreten. Eine wachsende Sprossachse krümmt sich bei einseitiger Beleuchtung so lange, bis die wachsende Spitze in der Richtung des Lichtstrahls steht; während selbstverständlich eine Progressivbewegung in der Richtung des Lichtstrahls, welche bei frei beweglichen Pflanzen und Thieren die Regel ist, bei festsitzenden Organismen unmöglich ist. Jeder Laie, der im Zimmer Blumen gezüchtet hat, dürfte die beliotropischen Krümmungen bei Pflanzen aus eigener Anschaung kennen. Es fragt sich nun, ob bei festsitzenden thierischen Organismen im Falle einseitiger Belenchtung ebenfalls diese heliotropischen Krümmungen eintreten. Ich will im Folgenden zeigen, dass das thatsächlich der Fall ist.

rischer Heliotropismus). Sitzungsberichte der Würzburger physik.-med. Gesellschaft 1888. Groom u. Loeb, Der Heliotropismus der Larven von Balanus perforatus und die periodischen Tiefenwanderungen pelagischer Thiere. Biolog. Centralblatt 1890, Bd. X. 
Weitere Untersnchungen über den Heliotropismus der Thiere etc. 393

I.

1. Ich stellte meine Versuche an einem grösseren marinen Ringelwurm, Spirographis Spallanzanii, an. Derselbe lebt in einer Röhre, welche zwar sehr biegsam, aber doch hinreichend starr ist, um das Thier in einer bestimmten Orientirung dauernd $\mathrm{zu}^{\mathrm{er}}$ halten. Diese Röhre wird durch Drüsensekretionen des Thieres gebildet und das aborale Ende der Röhre wird (ebenfalls durch ein Sekret) an Felsen oder anderen festen Körpern angeheftet. Aus dem anderen offenen Ende der Röhre ragen für gewöhnlich nur die Kiemen des Thieres hervor, die an der Spitze des Kopfes in mehreren spiraligen Windungen radiär zur Längsaxe des Kopfes angeordnet sind. Da die Röhre fast undurchgängig ist für Lichtstrahlen, so wirkt das Licht wesentlich nur auf den radiären Kiemenkranz des Thieres ein. Augen besitzt das Thier, so viel bis jetzt bekannt ist, nicht.

Das Thier ist in der Röhre, die innen völlig glatt ist, frei beweglich und kann ohne die geringste Verletzung, wenn man die Röhre aufschneidet, aus derselben herausgenommen werden. Ich habe anch, wenn das Wasser im Aquarium schlecht wurde, gelegentlich den Wurm die Röhre spontan verlassen lassen.

Der Laie, der die in der Röhre steckenden Thiere betrachtet, wenn sie ihre Kiemen voll entfaltet haben, glaubt zunächst Pflanzen vor sich zu haben, die an einem langen nackten Stamm (der Röhre) eine palmenartige Krone (die Kiemen) tragen. Bei einer leisen Erschütterung aber ziehen die Thiere ihre Kiemen pfeilschnell in die Röhre zurück. Dagegen habe ich eine Bewegung der Röhre durch die Thiere niemals unmittelbar wahrgenommen.

Wenn man ein ans der See entnommenes Thier in ein Aquarium bringt, so verhält sich das Thier zunächst gleichgilitig gegen das Licht. Das dauert so lange, bis das Thier das Fussende der Röhre am Boden festgeheftet hat, worüber oft mehrere Tage vergehen. Sobald das aber geschehen ist, beginnt der orientirende Einfluss des Lichtes sich bemerkbar zu machen. Fällt das Licht nur von einer Seite her anf das Thier, so treten bei ihm beliotropische Krümmungen der Röhre ein. Es wendet seinen oralen Pol zur Lichtquelle and krümmt seine Röhreso lange, bis die Axe seines Kiemenkranzes in 
die Richtung der Lichtstrahlen fällt. In dieser Orientirung verharrt das Thier alsdann dauernd, vorausgesetzt, dass die Richtung der Lichtstrahlen nicht geändert wird.

2. Um genauer zu prüfen, inwieweit die Richtung der Lichtstrahlen bestimmend ist für die Orientirung der Thiere, setzte ich dieselben in ein Aquarium, welches am Fenster stand und das durch einen Zinkkasten völlig verdunkelt werden konnte. Die Conturen des Aquariums sind in der Zeichnung (Fig. 1 und 2), welche dieser Abhandlung beigegeben sind (Taf. IX), mit vollständigen Linien, die Conturen des Zinkkastens mit unterbrochenen Linien gezeichnet. Die eine schmale Wand $a, b, c, d$ des Zinkkastens war in verticaler Richtung verschiebbar, und mittelst dieses Schiebers wurde der Lichteinfall ins Aquarium modificirt. Der Zinkkasten, dessen Wände innen geschwärzt waren, wurde so über das Aquarium gestülpt, dass der Schieber auf der Fensterseite des Aquariums sich befand. War der Schieber weit heruntergelassen, wie es in Fig. 2 der Fall ist, so fielen die Strahlen nahezu borizontal ein. War der Schieber weiter in die Höhe gezogen (wie in Fig. 1), so fielen neben den horizontalen auch noch von oben her Strahlen des Himmelslichtes ein, welche der Intensität nach stärker waren als die horizontal einfallenden Strahlen, weil sie nicht, wie diese, durch die im Wege stehenden Objecte (Bäume, Häuser) in ihrer Intensität reduzirt waren.

Ich legte am 14. XII. 899 kräftige ca. $15 \mathrm{~cm}$ lange Exemplare von Spirographis Spallanzanii auf den Boden des Aquariums mit der Längsaxe ihrer Röhre senkrecht gegen die Ebene des Fensters. Acht davon lagen mit dem oralen $\mathrm{Pol}$ nach der Zimmerseite $e, f, g, h$ (Fig. 1) des Aquariums, 1 nach der Fensterseite desselben. Die ersten zwei Tage vergingen, ohne dass eine Aenderung der Orientirung erfolgte; die Thiere hefteten zunächst das aborale Ende ihrer Röhre am Boden des Aquariums fest. Dann, im Laufe des dritten Tages, begannen sich die Röhren bei sechs von den Thieren, welche mit dem oralen $\mathrm{Pol}$ gegen die Zimmerseite gerichtet waren, in einer nahezu horizontalen Ebene zu krümmen, wobei die Conkavität der Krïmmung gegen das Fenster gerichtet war. Die beiden anderen Thiere, die sich ebenfalls mit dem Kopf gegen die Zimmerseite gelegt hatten, erhoben erst ihren Kopf und krummten dann die Röhre ebenfalls konkar gegen das Fenster. Das neunte Thier endlich, wel- 
Weitere Untersuchungen über den Heliotropismus der Thiere etc. 395

ches ich mit dem Kopfe gegen das Fenster ins Aquarium gelegt hatte, erhob nur den Kopf ein wenig.

In den nächsten Tagen begannen die 6 zuerst erwähnten Thiere ihren Kopf ein wenig mehr aufzurichten, so dass die Thiere am 22. XII., also 8 Tage nachdem sie in das Aquarium gelegt waren, sämmtlich in der gleichen Weise gegen das Licht orientirt waren. Der Kopf war gegen das Fenster gerichtet und die Symmetrieaxe des dem Lieht ausgesetzten Kiemenkranzes stand in der Richtung der vonaussen and oben fallenden intensiveren Strahlen des Himmelslichtes. Ich wartete nun ah, ob diese Orientirung eine dauernde sein würde. Bis zum 16. Februar 1890, also uiber 2 Monate, blieb das Aquarium nnverändert: die Thiere blieben ebenfalls unverändert in der in Fig. 1 wiedergegebenen Stellung.

3. Am Nachmittag des 17. Februar 1890 wurde das Aquarium um eine verticale Axe um $180^{\circ}$ gedreht und der Zinkkasten wieder so tiber das Aquarium gestiulpt, dass die verschiebbbare Wand wieder gegen das Fenster gerichtet war. Durch die Umdrehung des Aquariums war bewirkt worden, dass der bis dahin nach der Lichtquelle gerichtete Kopf der Thiere plötzlich der Zimmerseite des Aquariums zugekehrt war. Durch diese Umdrebung des Aquariums sollte ermittelt werden, ob bei einer Aenderung der Richtung der Lichtstrablen die Thiere die heliotropische Krïmmung rückgängig machen und ihren Kopf wieder der Lichtquelle zukehren würden. Im Laufe des Nachmittags und in der Nacht änderte sich im Aquarium nichts. Aber gegen Mittag des folgenden Tages fand ich zwei Thiere, welche sich am Morgen noch in der Stellung $A B$ (Fig. 3a) befunden hatten, in der Stellung $A B_{1} ; F$ bedeutet dabei die Ebene des Fensters. Das Stück $D B$ der Röhre hatte um den Punkt $D$ als Centrum die Fläche $D B B_{1}$ beschrieben. In dem gleichen Sinne trat an demselben und im folgenden Tage eine Aenderung der Orientirung aller ibrigen Thiere ein. Bei diesem Versuche war aber in Bezug anf die Richtung der Strahlen noch ein zweiter Umstand modificirt worden; nämlich die Wand $a b c d$ war weiter heruntergelassen, so dass fast nur horizontale Strahlen ins Aquarium einfielen (Fig. 2). Ich wollte damit ermitteln, ob nunmehr auch die Thiere der Richtung der Lichtstrahlen wieder folgen und sich ebenfalls nahezu horizontal einstellen würden. Das trat in der 


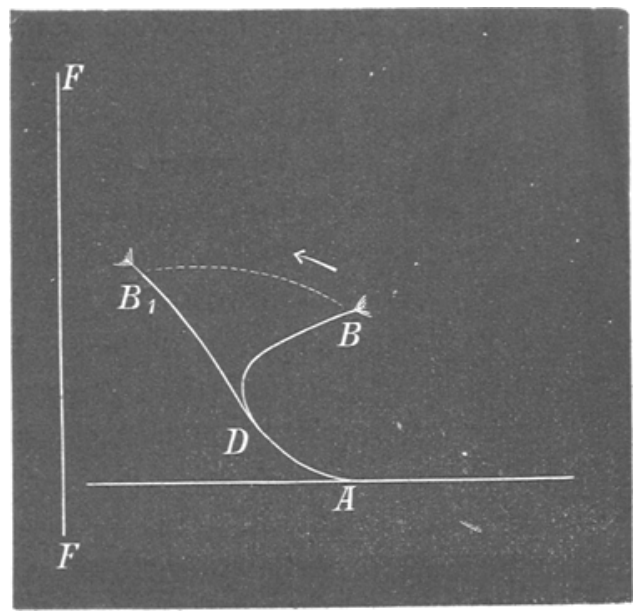

Fig. $3 a$.

That ein. An 22. II. 1890, also 5 Tage nach der Umkehr, war die Orientirung erreicht, welche in Fig. 2 wiedergegeben ist. Die Thiere haben die Köpfe gegen die Lichtquelle gewendet und die Axe ihres Kiemenkranzes steht in der Richtung der Lichtstrablen nahezu horizontal. Bis gegen Ende März liess ich die Bedingungen des Versuches unverändert, und bis dahin blieben die Thiere auch in der gleichen Orientirung.

4. Fallen die Lichtstrahlen vertical von oben in das Aquarium, so richtet anch die Spirographis ihre Röhre vertical aufwärts, so dass sie in ihrer Orientirung vollkommen einem im Freien vertical wachsenden Spross gleicht. Ich stellte diesen Versuch in einem anderen Aquarium an, in welches die Lichtstrahlen wesentlich nur von oben her einfielen. Man findet die Thiere meist auch in dieser Stellung im Schauaquarium der zoologischen Station in Neapel, in welches das Licht wesentlich von oben ber einfallt; nur dass hier, wo freischwimmende Thiere die Orientirung der Spirographis leicht stören, die Orientirung nicht immer eine so präcise ist, wie bei Vermeidung aller störenden Nebenumstände in besonderen Versuchsaquarien.

5. Aus den vorhergehenden Versuchen folgt, dass die Schwerkraft, wenn sie mit dem Licht zusammen auf die Thiere wirkt, nur einen minimalen Effekt auf die Orientirung der Thiere erzielen kann. Es musste noch untersucht werden, ob in einem vollkommen dunkeln Raum unter dem Einfluss der Schwerkraft 
Weitere Untersuchungen über den Heliotropismus der Thiere etc. 397

allein nicht etwa eine geotropische Aufrichtung der Thiere zu Stande komme.

Ich legte am 21. III. 1890 eine grössere Zahl von Spirographis horizontal auf den Boden eines im Dunkelzimmer stehenden Aquariums: Am 24. III. hatten die Thiere das aborale Ende der Röhre meist befestigt. Dann wurde das orale Ende der Röhre soweit erhoben, dass die Kiemen den Boden nicht mehr berïhrten. Die Axe der Spirale stand dabei nicht etwa vertical (wie bei verticalem Einfall der Lichtstrahlen, oder wie es hätte der Fall sein mïssen, wenn das Thier geotropisch reizbar wäre), sondern sie bildete einen sehr kleinen Winkel mit dem Horizont. So blieben die Dinge bis zum Ende des Versuches, der Mitte April abgebrochen wurde. Die Sehwerkraft bat also auf die Orientirung von Spirographis Spallanzani keinen wesentlichen Einfluss.

6. Die Contactreizbarkeit der Kiemen der Spirographis äussert sich darin, dass dieselben von festen Flächen weg gekrümmt werden. Diese Art der Reizbarkeit kann das Resultat bei heliotropischen Versuchen an diesen Thieren beeinflussen. Ich hatte mehrere Exemplare dieser Thiere auf den Boden eines Aquariums gelegt, das so flach war, dass die Thiere sich nicht aufrichten konnten. Die Spirographis wurde so in das Aquarium gelegt, dass ihre Längsaxe senkrecht zur Seite $a b$ (Fig. 3 b) des Aquariums lag und dass das Fussende $M$ der Röhren die Glaswand $a b$ beribhrt. Diese Wand $a$ war dem Fenster zugewendet und der Ebene desselben parallel. Die Thiere hefteten den Fuss an der Wand $a b$ fest und begannen darauf die ibnen

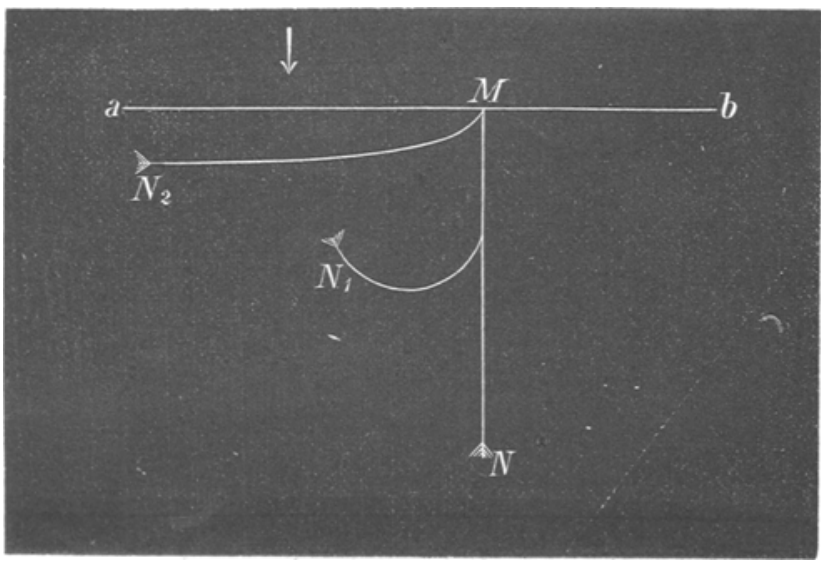

Fig. 3b. 
eigenthümliche heliotropische Krümmung anszuführen, wobei der Kopf der Lichtquelle zngekehrt wurde und die Röhre gegen die Lichtquelle konkav wurde. Die Röhre $M N$ ging in die Lage $M N_{1}$ über. Sobald aber bei fortschreitender Krümmung die Tentakel die Glaswand $a b$ berïhrten, wurde die Spitze $N_{1}$ ein wenig von der Glaswand weggebogen. Die heliotropische Krümmung erstreckte sich nun der Reihe nach auf alle Elemente der Röhre $M N$, so dass endlich die Spirographis in die Lage $\boldsymbol{M}$ $N_{2}$ gerieth, in welcher sie während der Beobachtungsdauer von 4 Monaten auch blieb.

Ich habe den gleichen Versuch mit dem gleichen Erfolg mehrere Male angestellt.

7. Die heliotropischen Erscheinungen bei Spirographis traten sowohl im directen Sonnenlicht, wie im diffusen Tageslicht ein. Die Lichtintensität, welche eben noch ausreicht diese Erscheinungen auszulösen, kann sehr gering sein. Ich habe noch nicht untersucht, wie die Strablen verschiedener Brechbarkeit sich bei diesen Thieren hinsichtlich ibrer Wirksamkeit verhalten. Da aber bisher für Pflanzen sowohl wie für Thiere die stärker brechbaren Strahlen sich als die heliotropisch wirksameren erwiesen haben, so ist zu vermuthen, dass auch Spirographis keine Ausnahme machen wird.

8. Sachs hat bekanntlich das Gesetz aufgestellt, dass radiäre Pflanzen orthotrop sind, d. h. ihre Längsaxe in die Richtung der Lichtstrablen resp. der Schwerkraft stellen. Es wird dem Leser nun aufgefallen sein, dass Spirographis, deren Körper, wie der aller Anneliden, dorsiventral und nicht radiär gebaut ist, sich gegen das Licht verhält wie ein radiäres Pflanzenorgan. Ich habe aber schon hervorgehoben, dass nur die radiär angeordneten Kiemen des Thieres dem Licht exponirt sind, während das übrige Thier in der Röhre dem Licht entzogen ist. Es zeigen also die hier mitgetheilten Beobachtungen, dass ein radiäres thierisebes 0rgan ebenfalls dem von Sachs fit die Pflanzen aufgestellten Orientirungsgesetz unterliegt (obwohl doch Spirographis ein Centralnervensystem besitzt, und die Pflanze nicht).

Es ist ferner von physiologischem Interesse, dass gerade das A th m u ng s organ der Spirographis von so hoher Lichtempfindlichkeit ist, dass die räumliche Orientirung des ganzen Thieres 
Weitere Untersuchungen über den Heliotropismus der Thiere etc. 399

wesentlich von dieser Lichtempfindlichkeit abbängt. Dieser Umstand kann vielleicht später eine andere Eigenthümlichkeit begreiflich machen, dass Branchiomma, eine der Spirographis in der Structur sehr ähnliche Serpulide, an seinem Kiemenblatt hochentwickelte Augen besitzt.

9. Nimmt man die Spirographis vorsichtig aus ihrer Röhre heraus, so ist sie ausser Stande, ihren Körper vom Boden aufzurichten. Sie kriecht dann, ähnlich wie ein Regenwurm, nur viel träger umber. Ich habe solche Thiere gelegentlich an die Fensterseite des Aquariums kriechen sehen. Sie schienen jedoch durch die Contactreize, denen sie in diesem Zustand fortwährend ausgesetzt sind, zu leiden; sie gingen in einigen Tagen alle zu Grunde.

10. Ueber den mechanischen Vorgang, durch welchen die heliotropische Kriimmung der Röhre bei diesen Thieren zu Stande kommt, vermag ich einstweilen keinen Aufschluss zu geben. Die Wand der Röhre eines ausgewachsenen Thieres erreicht eine Dicke von etwa $1 / 4-1 / 2 \mathrm{~mm}$ oder dariber. Sie ist sehr biegsam und elastisch. Nimmt man ei n. Thier, nachdem die Röhre heliotropisch gekrümt ist, ans der Röbre heraus, so behält die Röhre dennoch $\mathrm{d}$ a u e rnd d i e $\mathrm{K}$ r ù mmung bei. Es muss also die Wand auf der nach aussen konkaven Seite der Röhre dauernd verkürzt sein. Man könnte denken, dass die Elastizitätsgrenze der Röbre so niedrig liege, dass ähnlich wie bei einem Bleistab, die Röhre die ihr durch die Muskelkraft des Thieres ertheilte Kriimmung beibehielte. Das ist nicht der Fall. Ich fuhrte in eine gerade Röhre von Spirographis einen dicken Bleistab und bog ihn dann so, dass die Röhre stark gekrümmt wurde und liess den Bleistab in der Röhre. Als ich nach 8 Tagen den Bleistab aus der Röhre herauszog, behielt die Röhre nur eine Spur der ihr aufgedrungenen Krümmung bei. Ebensowenig gelang es mir, in dieser Weise eine heliotropisch gekrümmte Röhre wieder gerade zu machen; wie ich aber schon angedeutet habe, macht die Spirographis ihre gekrümmte Röhre bei passender Aenderung der Richtung der Lichtstrahlen in wenigen Stunden wieder gerade, und zwar so, dass die Röhre jetzt dauernd gerade bleibt. Auch die aufgeschnittene Röhre behält ibre Krümmung bei. Es steht aber dem Thiere ausser der unmittelbaren Anwendung von Druck und Zug noch 
ein anderes Mittel zur Verfugung, die Orientirung der Röhre dauernd zu ändern: nämlich die Ausscheidung von Secret und die Neubildnng einer inneren Schicht der Röhre. Der Gedanke, dass die Beständigkeit der Krümmung auf diesem Wege erreicht wird, wird unterstïtzt durch die Thatsache, dass die innerste Schicht der Röhre viel elastischer ist als die äusseren Schichten, so dass durch einseitige Neubildung einer inneren Schicht die Röhre bleibend gekrïmmt werden kann. Das geht aus folgenden Umständen hervor: Schneidet man eine Röhre der Länge nach auf, so rollen sich die Sehnittränder stark nach innen ein. Löst man - was leicht gelingt - die einzelnen Schichten der Röhre $a b$, so sieht man, dass diese Einrollung bei den inneren Schichten stärker ist als bei den äusseren und am stärksten bei der innersten, jüngsten Schicht. Es würde also die Neubildung einer inneren Schicht auf einer Seite der Röhrenwand genügen, um die Röbre dauernd gekrümmt zu erhalten. Unmittelbar lässt sich eine solche Neubildung nicht beobachten. Auch die Erwartung, die eine Seite der Röhrenwand dicker zu finden als die andere, wurde durch den Umstand getäuscht, dass in der Dicke der Wand völlig gerader Röhren an verschiedenen Stellen desselben Querschnitts die grössten Differenzen vorhanden waren, so dass die Dickenverhältnisse der Wandung gar nicht als Kriterium für unsere Frage zu benutzen waren. Ich kann deshalb die folgende Vermuthung über das Zustandekommen der heliotropischen Krümmung der Röhre nur unter dem Vorbehalt mittheilen, dieselbe später von Neuem zu prïfen und event. za berichtigen. Ich denke mir, dass bei einseitiger Beleuchtung das Thier zunächst die Axe des Kiemenblattes möglichst in die Richtung der Lichtstrahlen zu bringen sucht und dabei möglicherweise die Röhre mit Hülfe seiner Muskelkraft krümmt. Da aber die Röhre vermöge ibrer Elastizität ihre ursprïngliche Stellung wieder einzunehmen sucht, so muss sich der Körper der Spirographis an der inneren Wand der Röhre stärker reiben. Diese stärkere Reibung bedingt eine stärkere Ausscheidung des Secrets der Hautdrüsen, welches das Material der Röhre bildet. Dass in der That die Reibung zur Ausseheidung des Hautsekrets und zur Bildung der Röhre führt, habe ich bei einer Aktinie (Cerianthus membranaceus) direct nachweisen können. Für Spirographis habe ich Folgendes konstatiren können, was auf ähnliche Verhältnisse hinweist. Ich schnitt 
Weitere Untersuchungen über den Heliotropismus der Thiere etc. 401

kleine Stiicke aus der Röhre heraus. Infolge dessen musste das Thier bei seinen Bewegungen sich an den Schnittränderu reiben und in der That fand hier in kurzer Zeit eine starke Ausscheidung von Secret statt, so dass die Oeffnung bald durch eine neugebildete Membran geschlossen war. Zweitens findet eine Reibung beständig an dem vorderen Rand der Röhre statt, wenn das Thier den Kopf herausstreckt. In der That findet auch hier ein ständiges Längenwacbsthum der Röhre statt, wie man aus Fig. 4 ersehen kann. Ich hatte in diesem Falle aus der Röhre bei $a$ ein langes und breites Stiuck $a$, herausgeschiitten, so dass das vorderste Stück der Röhre $a, b$ nur durch ein dünnes Stück $p$ mit der übrigen Röhre zusammenhing. Das Thier steckte von nun an seine Kiemen bei $a$ heraus und benutzte den Theil $a, b$ der Röhre nieht weiter. In wenigen Tagen wurde neue Substanz bei $a$ angesetzt und im Lauf von 3 Wochen war das Stïck $a c$ neugebildet, das sich auch schon durch seine helle Farbe als neugebildet charakterisirte. Gleichzeitig hatte ich das aborale Ende der Röhre bei $d$ völlig abgeschnitten. Vor meinen Augen kam es bei dem Hingleiten des aboralen. Endes des Thieres auf dem Sande zu einer Ausscheidung eines klebrigen Hautsekrets, an dem die Sandpartikel hafteten. Auf diese Weise bildete sich das neue Röhrenstück $d e$, bestehend aus den durch das Drüsensecret der Röhre mit einander verkitteten Sandtheilen. Im Inneren war das neugebildete Röhrenstück völlig glatt. Die Sekretion der Hautdrüse findet eben so lange statt, bis keine nennenswerthe Reibung mehr vorhanden ist. - Wenn ich Spirographis aus dem Gehäuse heransnahm und in ein glattes Reagenzglas brachte, so fand so gut wie keine Sekretion statt. Nur aus den Parapodien wurde in Form langer feiner Fäden ein Sekret ausgesehieden, ähnlich dem aus den Spinndrüsen der Spinnen bervorgehenden. Wurde aber die aus dem Geläuse befreite Spirographis auf Sand gelegt, so bildete sich zunächst die Hülle am aboralen Ende aus den Sandpartikeln. Eine vollkommene Röhrenbildung aber habe ich bei solchen ganz aus dem Gehäuse befreiten Thieren nicht beobachtet, da sie alle bald starben. 


\section{II.}

Spirographis Spallanzanii erreichte ihre heliotropische Orientirung bei einseitiger Beleuchtung durch Krümmung ihrer biegsamen Röhre; ein Zuwachs der Röhre war dabei nicht nöthig. Nun gibt es andere Serpuliden, deren kalkhaltige Röbre starr und unbiegsam ist. Diese Serpuliden exponiren wie Spirographis dem Licht ihr Kiemenblatt, welches auch hier wieder seiner Struktur nach wie ein radiäres Organ reagiren muss. Es muss deshalb eine solche Serpulide, wenn sie heliotropisch reizbar ist, die Längsaxe ihrer kreiscylindrischen Röhre in die Richtung des Lichtstrahls stellen. Bringt man die Kalkröhre in eine andere Orientirung gegen den Lichtstrahl, so bleibęn dem Thier nur zwei Möglìchkeiten, die richtige Orientirung wiederzugewinnen: entweder die Kalkröhre zu verlängern und den neu hinzuwachsenden Theil so lange zu krümmen, bis die Symmetrieaxe der Röhre wieder in der Richtung des Lichtstrahls steht, oder die Röhre ganz zu verlassen und eine neue in der gesetzmässigen Orientirung zu bauen. Das erstere trifft zu. Ich stellte Versuche an Serpula uncinata an. Diese Anneliden bewohnen kalkhaltige Röhren und leben gesellig. Man findet im Golf von Neapel grosse weisse Blöcke, die lediglich aus den Röhren unzähliger solcher aneinandergefügter Wurmröhren bestehen. Es fiel mir an solchen Blöcken auf, dass die einzelnen Röhren alle, gleiche Richtung hatten und zwar liess sich bei Blöcken, an welchen die Basis, mit der sie auf dem horizontalen Boden auflagen, deutlich zu erkennen war, ersehen, dass die Röhren mit der Längsaxe vertikal im Wasser gestanden haben mussten. Die Serpulen können sich in der Röhre, ähnlich wie die Spirographis, frei bewegen. Ich legte vun einen grossen Block, der aus zahlreichen Wurmröhren bestand und dessen einzelne Röhren alle fast mathematisch genan geradlinig und parallel waren, so auf den Boden des Aquariums, dass die Längsaxe $a b$ der Röhre, welche vorher vertikal stand, jetzt eine horizontale Lage erbielt (Fig. 5). Das Licht fiel von oben her ins Aquarium. Im Laufe der nächsten Tage bemerkte ich, dass die Serpulen, welche, wie Spirographis, nur ihre radiär angeordneten Kiemen dem Lichte darbieten, dieselben stark zenithwärts bogen. Dann begannen einzelne Röhren zu wachsen und zwar in der Weise, dass die neu binangewachsenen 
Weitere tntersuchungen über den Meliotropismus der Thiere etc. 403

Stücke der Röhren alle deutlich sich aufwärts $\mathrm{krumm-}$ ten und zwar so lange, bis die Spitze der Röhre die Richtung der Lichtstrahlen (die in diesem Falle mit der Ricbtung der Schwerkraft zusammenfiel) erreicht hatte. Von da ab wuchsen die Röhren in der Richtung der Lichtstrahlen (und der Schwerkraft) weiter. Nach 6 Wochen war der Block mit aufwärts gekrümmten Röbren ibersäet, während nicht eine einzige Röhre in der urspringlichen Richtung $a b$ weiter gewachsen war. Die Zeichnung giebt diese Aufwärtskrlimmung am freien Rand wieder. Es ist also hier der $\mathrm{S}$ a $\mathrm{chs}$ 'schen Theorie des Geotropismus und Heliotropismus entsprechend der Endeffekt wieder der, dass die Axe der wie ein radiäres Organ reagirenden Kiemen in die Richtung der Lichtstrablen (und der Schwerkraft) gestellt wurde. Während aber dieser Effekt bei Spirographis (deren Röhre biegsam ist) durch Orientirungs-Aenderung der alten Röhre erreicht wird, wird der gleiche Fiffekt bei Serpula, deren Röbren unbiegsam sind, durch heliotropische Krummung des im Wachstbum begriffenen Theiles der Röhre crreicht.

In dem eben angefühten Versuch war die Richtung der Lichtstrahlen identisch mit der Richtung der Schwerkraft. Ich habe einstweilen noch nicht entscheiden können, ob nur das Licht die Orientirung der Röhren bestimmt, oder ob auch die Schwerkraft daran einen Antheil hat. Ich hoffe später noch Versuche dariiber anstellen zu können.

III.

1. Ich habe mich bemilht, noch andere Thiere aufaufinden, bei welchen heliotropische Krimmungen nur im wachsenden Theile derselben stattfinden. Diese Bemïhungen hatten Erfolg bei Hydroidpolypen. Stämme von Sertularia (polyzonias?) wurden nahe der Wurzel abgeschnitten und mit der Spitze verkehrt in Sand gesteckt, so dass das Schnittende jetzt nach oben lag. Die Stämme wurden an das Fenster gestellt, durch welches das Licht schräg von aussen und oben einfiel. Sie begannen zu re- 
generiren; aus der Schnittstelle wuchsen sowohl neue polypentragende Sprosse, als auch neue Wurzeln hervor ${ }^{1}$; aber während die neuen Sprosse zum Fenster hin and zenithwärts wuchsen, wuchsen die Wurzeln zimmerwärts und gegen den Boden. Die polypentragenden Sprossesind positiv, die Wurzeln negativ heliotropisch. Dass die negativ heliotropischen Elemente echte Wurzeln waren, liess sich anch dadurch zeigen, dass sie mit einem festen Körper in Contact gebracht an der Oberfläche desselben sich festhefteten und dicht an der Oberfläche weiter wuchsen. Nur mit Anwendung von Gewalt war es möglich, sie von der Oberfläche loszulösen. Die polypentragenden Sprosse haben diese Art von Contactreizbarkeit, sich an die Oberfläche fester Körper festzuheften, nicht. Die heliotropischen Erscheinungen lassen sich mit Hülfe der Figg. 6, $7 \mathrm{u} .8$ ohne viele Worte klar machen. $a b$ ist der alte Stamm, $b$ die Schnittstelle, bis $a c$ steckte der Stamm im Sande. Die an der Schnittstelle $b$ neugebildeten Wurzeln $W_{1}$ entspringeu aus der Schnittstelle und krümmen sich vom Licht fort gegen die Zimmerseite und zum Boden. Die neuentstehenden polypentragenden Sprosse $S$ wachsen aufwärts und zum Fenster. Der Pfeil giebt die Richtung der Strahlen des Himmelslichtes bei diesem Versuche an.

2. In diesen Versucheu entstanden gelegentlich mitten am alten Stamme neue Ausläufer, welche in Bezug auf ihre Contactreizbarkeit wie Wurzeln reagirten. Solche Ausläufer, welehe sich an festen Körpern anhefteten, waren stets negativ heliotropisch. Sie wuchsen abwärts und gegen die Zimmerseite und blieben frei von Hydranthen. Vgl. Fig. $6 W_{2}$.

Dagegen sah ich auch, wenngleich viel seltener, an den alten Stämmen neue polypentragende Sprosse entstehen; diese wuchsen in umgekehrter Richtung, nämlich zenithwärts.

3. Dass es in der That bei Sertularia nur der wachsende Theil ist, der die heliotropischen Krïmmungen ausfubrt, konnte ich durch folgenden Versuch konstatiren. Einer grösseren Zahl von Sertularienstämmen wurde die Spitze abgeschnitten. Dieselbe begann im Lauf von wenigen Tagen neu zu sprossen; der neu sprossende Theil ist in seiner Farbe auf-

1) Was für die Theorie der Organbildung von Bedeutung ist. 
Weitere Untersuchungen über den Heliotropismus der Thiere etc. 405

fallend rom alten Stamm verschieden; während dieser (durch aufgelagerte Algen?) bräunlich ist, ist die Farbe des neu sprossenden Theiles ein helles Gelb. Die nea sprossenden Elemente krümmten sich solange, bis die Spitze in die Richtung der Lichtstrablen gekommen war und wuchsen alsdann in der Richtung der Lichtstrahlen weiter. Am alten Theil des Stammes trat in dieser Zeit keine Aenderung der Orientirung ein, ebensowenig bei anderen unversehrt gelassenen Stämmen, an denen in dieser Zeit kein Längenzuwachs stattfand.

Inwieweit die Schwerkraft bei diesen Versuchen eine Rolle spielt, habe jch nicht genau feststellen können. Meine im Dunkelzimmer kultivirten Sertularien hörten auf zu wachsen; ob bloss infolge des Lichtmangels, bezweifle ich.

3. Der Einfluss des Lichtes (und event. der Schwerkraft) erstreckt sich nicht nur auf die Orientirung der neu gebildeten Organe, sondern auch auf den Ort der Organbildung. Ich habe (und nicht allein bei Sertularia) beobachtet, dass die neuen polypentragenden Aeste stets auf der zenithwärts gerichteten Seite des Stammes entstehen. So entsteht in Fig. 8 ein neuer Spross $S$ auf der zenithwärts (der Lichtquelle) zugekehrten Seite des Ausläufers $W_{1}$. Ich gehe auf diese Dinge hier nicht näher ein, da sie Gegenstand einer demnächst erscheinenden Arbeit uber die Form der Thiere sein werden.

Die hier mitgetheilten Versuche an Sertularia sollen nur dazu dienen, um das allgemeine Bild des thierischen Heliotropismus zu vervollständigen und die Thatsache der Identität des thierischen und pflanzlichen Heliotropismus zu erweitern. Dagegen ist die spezielle Erforschung der heliotropischen Verhältnisse bei Hydroidpolypen noch Aufgabe kommender Untersuchungen. Dass sie interessant and fruchtbar ist, zeigt die schöne eben erschienene Abhandlung von Hans Driesch: „Heliotropismus bei Hydroidpolypen“1), die ihn zu folgendem Resultat geführt hat: „Die unter ungünstigen Verhältnissen von Sertularella polyzonias an Stelle von Personen erzeugten Stolonen sind bis auf den ersten, von Anfang an sich rom Licht abwendenden, zuerst positiv und werden nach Erzeugung ihrer Tochterstolonen negativ heliotropisch. Sie entstehen an der dem Licht zugewendeten Seite des Mutterstolo"

1) Zoologische Jahrbüicher Bd. V. 
(S. 152). Die Beobachtung von Drieseh lässt sich mit meinen Beobachtungen sehr wohl vereinigen. Ich komme darauf in meiner Abhandlung zur physiologischen Morphologie der Thiere zurück.

Die Resultate dieser Abhandlung lassen sich in folgende Sätze zusammenfassen :

1. Gewisse festsitzende Thiere (Serpuliden, Hydroidpolypen), welche der Structur ihrer dem Licht exponirten Organe entsprechend wie radiäre Organismen auf Licht und Schwerkraft reagiren müssen, stellen die Symmetrie-Axe des radiären Organs dauernd in die Richtung des Lichtstrahls, wie radiäre festsitzende Pflanzenorgane.

2. Der Umstand, dass derartige festsitzende Thiere, wie die Serpuliden, ein centrales Nervensystem besitzen, die Pflanzen dagegen nicht, bedingt keinen Unterschied in dem heliotropischen Effekt.

3. Handelt es sich um einseitigen Einfall der Lichtstrahlen, so treten bei den erwähnten Thieren dauernde heliotropische $\mathrm{Kr}$ ümm ungen ein, wie bei festsitzenden pflanzlichen Organen im gleichen Falle.

4. Es giebt festsitzende Thiere, welche diese heliotropische Krümmung, wie viele Pflanzen, nur durch Wachsthum erreichen. Dahin gehören u. a. Sertularia und Eudendrium, bei welchen sich nur die im Wachsthum begriffenen Theile heliotropisch zu krümmen im Stande sind, und dahin gehört ferner Serpula uncinata, welche die Orientirung ihrer sonst starren Röhre nur in dem wachsenden Theil derselben zu verändern im Stande ist.

5. Spirographis Spallanzanii, deren Röhre biegsam ist, erreicht ihre heliotropische Krïmmung ohne Wachsthumserscheinung, wie auch gewisse mit Gelenken versehene Pflanzenorgane die heliotropische Orientirung ohne Wachsthumserscheinungen erreichen können. 
Weitere Cntersuchungen über den Heliotropismus der Thiere ctc. 407

Wenn ich mit dem hier Mitgetheilten auch meine Untersuchung ïber den Heliotropismus noch nicht für abgeschlossen halte, so glaube ich doch damit gezeigt zu haben, dass auch der Heliotropismus festsitzender Thiere im Wesentlichen durchaus identisch ist mit dem Heliotropismus festsitzender Pflanzen. Was den Heliotropismus frei beweglicher Thiere betrifft und was die Bedeutung angeht, welehe der Heliotropismus für die Lebensgestaltung der Thiere hat, so findet der Leser, der sich dafür interessirt, Näheres in meinen früheren Arbeiten.

\section{Anhang.}

Ueber den Instinkt und den Willen der Thiere.

1. In den biologisehen Wissenselaften giebt es noch immer Autoren, welche den "Instinkt" und den "Willen" der Thiere wie einen bewegungsbestimmenden Lmstand bebandeln, so dass der Naturforscher, wenn er in das Gebiet der belebten Natur eintritt, hier auf eine ganz neue Kategorie von Ursachen trifft, nämlich solche, die vor unseren Augen angeblich fortwährend grosse Effekte üben, ohne dass es möglich wäre, in der Technik von diesen Ursachen jemals Gebrauch zu machen. Der "Instinkt" und der „Wille" der Thiere steht als bewegungshestimmender Umstand auf derselben Stufe wie die ubernatürlichen Mächte der 'Theologen, die auch angeblich bewegungsbestimmende Umstände sind, auf die sich aber ein Techniker leider anch nie wuirde verlassen können.

Meine Untersuchungen iiber den Heliotropisnus der Thiere flibrten mich darauf, in einigen Fällen, in welchen nach bisheriger Annahme ein Thier "freiwillige" oder ,instinktive" Bewegungen ausfibrte, die Umstände näher zu analysiren, welche die jedesmalige anscheinend „zufällige“ Richtung der Bewegung näher bestimmen. Da, wo ich der Ursache solcher "freiwilligen" oder ,instinktiven" Bewegungen der Thiere nachging, stiess ich bis jetzt ausschliesslich auf solche Unstände, welche auch in der unbelebten Natur als bewegungsbestimmend bekannt sind, so dass es möglich ist, mit Htilfe dieser selben Ursachen die "freiwilligen“" Bewegungen eincs lebenden Thieres ebenso sicher und eindentig 
zu beherrschen, wie der Techniker bisher die Bewegungen der unbelebten Natur beherrscht hat. Was man für den Effekt des Willens oder des Instinktes hielt, das war in Wirklichkeit Effekt des Lichtes, der Schwerkraft, der Reibung, chemischer Kräfte u. s. f. Ich will das etwas näher ausführen.

Die Stellung, welehe die Röhre von Spirographis Spallanzani im Raume hat, ist, wie wir vorhin sahen, so, dass das Thier den oralen Pol dem Licht zukehrt und die Axe seines radiären Kiemenblattes in die Richtung der Lichtstrahlen stellt. Die Richtung der Lichtstrahlen ist der Umstand, welcher die Orientirung dieses Thieres eindeutig bestimmt. Wenn es in der Technik darauf ankäme, eine grosse Zahl von lebenden Spi-

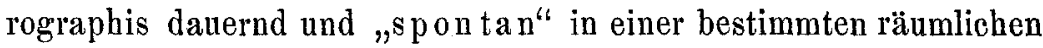
Orientirung $\mathrm{zu}$ erhalten, so würden unsere Untersuchungen angeben, wie man das zu machen hätte: es wäre nur nöthig, die Lichtstrahlen in der gewiinschten Richtung auf die Thiere fallen zu lassen. Versuche es doch Einer mit den "Instinkt" oder dem „Willen", die Spirographis zu einer bestimmten räumlichen Orientirung zu zwingen: er wird den Lichtstrahl zu Hülfe nehmen müssen, um zum Ziele zu gelangen; auch wenn er binterher glaubt, dass ausser, neben, vor, hinter oder zwischen dem Lichtstrahl noch der "Instinkt" oder der „Wille“ des Thieres als bewegungsbestimmender Umstand mit thätig gewesen sei. Er wird sich ferner überzengen, dass die Richtung des Lichtstrahls, falls genügende Intensität desselben vorhanden ist, alle in und eindeutig die Orientirung zu bestimmen im Stande ist.

Die Richtung der "freiwilligen" Bewegungen der geflügelten Blattläuse ist bestimmt durch die Richtung der Lichtstrahlen. Die Thiere sind gezwungen, den oralen Pol dem Licht zuzukehren und in der Richtung des Lichtstrahles sich zu bewegen. Bringt man die Thiere in ein durchsichtiges Gefäss, so leben und sterben sie an der dem Lichte zugekebrten Seite desselben. Wem es darauf ankäme, diese Thiere zu zwingen, sich in einer bestimmten Richtung zu einem bestimmten Ziele "freiwillig" zu bewegen, der weiss jetzt, wie er das zu machen bat: er lässt hinreichend intensives Licht in der gewünschten Richtung auf die Thiere fallen.

Die Richtung der Lichtstrablen, und zwar der stärker brechbaren, bestimmt bekanntlich auch die Orientirung pflanzlicher 
Gebilde. Der Botaniker lenkt mit Hülfe des Lichtstrahls die Orientirung pflanzlicher Organe so, dass sie seinen Zwecken entspricht. Welchen Sinn hätte es für ihn zu behaupten, dass ausser dem Lichtstrahl auch noch der „Wille“ oder der „Instinkt" der Pflanze mitwirkte, wenn die Orientirung schon durch das Licht alle in und e indeutig bestimmt ist? Das Abhängigkeitsverbältniss der thierischen Bewegungen rom Licht ist aber, wie ich gezeigt habe, Punkt für Punkt identisch mit dem Abhängigkeitsverhältniss der pflanzlichen Bewegungen vom Licht. Man hat mir aber trotzdem eingewendet, dass für die Orientirung der Pflanzen bei passender Versuchsanordnung zwar das Licht allein bestimmend sei, bei den Thieren aber, bei denen es ja allerdings auch bei passender Versuchsanordnung allein und eindeutig bestimmend ist, sei doch noch der Instinkt und der freie Wille mit bestimmend.

Wie in den eben erwähnten und in vielen anderen in meiner Abhandlung über Heliotropismus angeführten Beispielen die Richtung der Lichtstrahlen (und zwar insbesondere der stärker brechbaren) der wesentliche Umstand ist, welcher die Bewegungen der Thiere bestimmt, so ist es in anderen Fällen die Schwerkraft, in wieder anderen der Contakt fester Körper, in noch anderen chemische Kräfte $\mathfrak{u}$. s. f.

2. Um in jedem Falle "freiwilliger" Bewegung eines Thieres die Ursache mit anzugeben, welche die Bewegung bestimmt, bezeichnete ich die Bewegungen nach dieser ibrer äusseren Ursache; da es uns ja darauf ankommt, diese „freiwilligen" Bewegungen zu beherrschen. Ich sprach deshalb, wie es in der Pflanzenphysiologie schon lange geschieht, von Heliotropismus, wenn die Richtung des Lichtstrahls dem Thiere die Richtung der Bewegung oder die Orientirung vorschreibt, von Geotropismus, wenn es die Schwerkraft ist, von Stereotropismus, wenn der Contakt fester Körper die Orientirung oder die Bewegung bestimmt $u$. s. f.

Ein Zoologe warf mir vor, was denn damit gewonnen séi, wenn ich das als Stereotropismus bezeichne, was man bisher als Instinkt bezeichnet habe. Es handelte sich um die Erscheinung; dass gewisse Thiere sich in den Spalten fester Körper verkriechen und jener Zoologe meinte, dass die Thiere das eben "aus Instinkt" thun. Wenn der Physiker die Beobachtung macht, dass Flüssigkeiten in einer Capillare aufsteigen oder dass die eine Flïssigkeit in einem Glasrobr einen konvexen, die andere einen konkaven 
Meniskus bildet, so wird er weniger genügsam sein als jener Zoologe, für den alles ,aus Instinkt" geschieht. Der Physiker wird nach den näheren Umständen, welche die Erscheinung bestimmen, suchen. Das Gleiche scheint mir auch die Aufgabe eines Naturforschers den Lebenserscheinungen gegenüber zu sein; eine A ufgabe, die dureb die Bezeichnung, dassei, ,Instinkt", gar nicht einmal erkannt, geschweige den n g elöst wird. So lag es vom naturwissenschaftlichen Standpunkt aus zunächst nahe anzunehmen, dass das Licht es sei, welches die Thiere bestimme, in die Spalten sich zu verkriechen. Allein ich konnte feststellen, dass die Thiere auch dann in die Lücken zwischen festen Körpern eindringen, wenn man völlig durehsichtige Körper wählt und diese dem vollen Licht aussetzt; und zweitens, dass sie es auch dann noch thun, wenn sie sicb in einem völlig finsteren Raume befinden. Das Licht ist also nicht die physikalische Ursache, welche jene Erscheinung bestimmt. Ich stellte das für Forficula, Ameisen, Larven von Musca vomitoria u. a. fest. Vor mir hat Plateau ein ähnliches Verhalten fuir Cryptops festgestellt, was mir damals noch unbekannt war. Die Ursache für dieses Eindringen der Thiere in enge Räume ist also nicht das Licht, sondern der allseitige Contakt mit festen Körpern und zwar der dadurch erzeugte Druck und die Reibung. Was diese Einsicht, dass das Licht mit dieser Erscheinung nichts zu thun hat, sondern dass es die durch den Contakt fester Körper erzengte Reibung resp. Druck ist, vor der Phrase, dass es der Instinkt sei, voraus hat, ist der Umstand, dass Druck und Reibung physikalische Agentien sind, die wir, wie das Licht, quantitativ und qualitativ beherrschen und mittelst deren wir dem Thier seine "freiwillige" Bewegung oder seine ,freiwillige" Orientirung eindeutig vorschreiben können. - Ich will nun bei dieser Gelegenheit noch mittkeilen, dass, wie es eine grosse Zahl von Thieren giebt, welche gezwungen sind, ihren Körper möglichst allseitig mit festen Körpern in Contakt zu bringen, es auch andere gibt, welche die gerade entgegengesetzte Art von Reizbarkeit zeigen und von jedem festen Körper, mit dem sie zufällig in Contakt kommen, sich sofort zurïckziehen. Dahin gehören die Nauplien von. Balanus perforatus, die kleinen Mysiden des Golfes von Neapel, das Kiemenblatt von Spirographis Spallanzani u. s. f. Dass aber die von 
Weitere Lntersuchungen über den Heliotropismus der 'Thiere etc. 411

mir als Stereotropismus bezeichnete Form von Reizbarkeit eine hervorragende Rolle in den Lebenserscheinungen spiclt, geht schon daraus hervor, dass das Eindringen der Spermatozoen ins Fi, wie Untersuchungen von $\left.D e w i t z^{1}\right)$ zeigen, von dieser Art von Reizbarkeit ahbängt und dass ferner auch dic Wanderungeu der Lcukocythen in hervorragender Weise durch diese Contaktreizbarkeit bestimmt werden. - Ich habe ferner inzwischen bei Intersuchungen über den Einfluss äusserer Reizursachen anf die Körperform gefuuden, dass dieser Stereotropismus sowohl die Form, als auch die Grösse und Schnelligkeit des Wachsthums gewisser Organe beeinflusst. Es handelte sich un Versuche an Ifydroidpolypen. Was die Form betrifft, so gelang es mir, st e reotropische Kriummugen (vom festen Körper fort) mit derselben Sicherbeit bei gewissen Organen zu erzielen, wie es mir gelungen ist, heliotropische Krümmungen bei Thieren zn erziclen. Was die Schnelligkeit und die Grösse des Längenwachsthums betrifft, so erreichen gewisse Organe, wenn sie frei wachsen, in der gleichen Zeit bei sonst völlig gleichen Bedingungen nur den zebnten Theil der Länge, den sie erreichen, wenn sie sich in einseitiger Berührung mit einem festen Körper befinden. Ich glaube also durchaus keinen Missgriff und durehans nichts Unnitzes gethan zu baben, wenn ich auf die Belleutung diesel Contaktreizbarkeit im Thierreich aufmerksam gemacht und es für nöthig befunden habe, sie mit cinem besonderen Namen zu belegen.

3. Ich babe bisher Beispicle angeführt, in welchen eine einzige Reizursache die freiwilligen Bewegungen der Thiere bestimmte. Allein in einer grossen Zahl von Fällen sind die Bewegungen der Thiere nicht nur abhängig bloss von ciner einzigen Reizursache; es wirken vielmehr häufig mehrere Ursachen glcichzeitig, und die auftretende Bewegung, deren Ursache dann wieder der "Wille" sein sollte, ist eben nur die resultireude der verschiedenen gleichzeitig auftretenden Ursachen. Bei sebr intensivem Lichte geht die ausgewachsene Larve von Musca vomitoria in der Richtung des Lichtstrahls vom Lichte fort; sie geht dicht an einem neben ihrem

1) Dewitz, Leber die Vereinigung der Spermatozocn mit dem Ei. Pflüger's Archiv Bd. 37. - Vgl. auch Massart, Sur l'irritabilité des spermatozö̈des de la grenouille. Bullet. de l'Académie Royale de Belgique Bruxelles 1888. (Wiederholung und vollständige Bestätigung der Versuche von Dewitz.) 
Weg liegenden Fleischstiuck vorbei. Ist das Licht hinreichend schwach, so überwiegt der chemische Einfluss der vom Fleischstück ausgehenden flüchtigen Bestandtheile den orientirenden Einfluss des Lichtes und die Larve gelangt an's Fleisch. Bei anderen Thieren, die noch viel empfindlicher sind gegen chemische Reize, wie z. B. die männlichen Schmetterlinge, die bekanntlich von einem Weibchen rein durch chemische Einflüsse aus grosser Entfernung angelockt werden, kann der Heliotropismus ganz verdeckt werden durch diese chemischen Reizwirkungen. Es ist nicht in jedem Falle einfach, aus der jedesmal resultirenden Bewegung die einzelnen bewegungsbestimmenden Umstände festzustellen.

4. Es tritt noch ein zweiter komplizirender Umstand hinzu. Die Lebenserscheinungen sind Reizerscheinungen, d. h. sie sind nicht allein abhängig von den in jedem Augenblick wirkenden äusseren Ursachen, sondern von diesen und von den im Innern des Thieres vorhandenen Umständen zusammengenommen, und diese letzteren Umstände sind selbst variabel. Die Untersuchungen über den Heliotropismus der Thiere ergaben, dass dasselbe Thier in verschiedenen Epochen seiner Existenz sich dem Licht gegenüber verschieden verbalten kann. Die überwinterten und ungefütterten Raupen von Porthesia chrysorrboea sind energisch positiv heliotropiseh; haben die Thiere gefressen, so ist der Heliotropismus zwar noch nachweisbar; aber intensives Licht, das frïher die Bewegung eindeutig bestimmte, wirkt jetzt nur mehr so, wie früher ganz schwaches Licht wirkte. Einige Zoologen haben auch im Anschluss an diese Beobachtnng meine Versuche bemängelt und bemerkt, dass doch Versuche an so armen verhungerten Thieren nicht recht beweiskräftig seien. Es kann sich aber doch Niemand die Thatsache aus den Fingern saugen, dass die Raupe von Porthesia chrysorrhoea gerade bei leerem Magen ausserordentlich lichtempfindlich und noch dazu positiv heliotropisch ist, und es ist doch für einen naturwissenschaftlich Denkenden nicht etwa ein Mangel meiner Versuche, dass ich nachgewiesen habe, dass die hungernden Raupen von Porthesia empfindlicher gegen Licht sind, als die gefütterten. Es wäre natürlich ausserordentlich erleichternd für unsere weitere Erkenntniss der Abhängigkeit thierischer Bewegungen vom Licht, wenn es uberall so wäre, dass im Hungerzustand ein Thier energisch heliotropisch wird; leider ist das nicht der Fall. Wenn die betreffenden Kri- 
Weitere Untersuchungen über den Heliotropismus der Thiere etc. 413

tiker meiner Arbeit sich die Mübe genommen baben würden, einige Seiten mehr in meinem Buch zu lesen, so würden sie gefunden haben, dass die Blattläuse gerade erst im gefütterten Zustand lichtempfindlich werden und zwar positiv heliotropisch, und dass die Larve von Musca vomitoria nur im völlig ausgefütterten Zustand energisch negativ heliotropisch wird u. s. f. Für die Ameisen hängt die Lichtempfindlichkeit, wie ich gezeigt habe, mit der Sexualität zusammen: die Männchen sind empfindlicher als die Weibchen, zur Zeit des Hochzeitsfluges tritt bei Männchen und Weibchen energischer Heliotropismus auf, während die sogenannten Arbeiterinnen davon so gut wie frei bleiben. Es kommen die Aenderungen im Sinne des Heliotropismus hinzu, der bei manchen Thieren in den verschiedenen Entwickelungsstadien stattfindet. So ist die ausgewachsene Larve von Musca vomitoria negativ heliotropisch, das geschlechtsreife Insekt dagegen positiv heliotropisch. Ein derartiges Verhalten ist sehr verbreitet.

Es finden sich endlich und nicht selten die Fälle, dass es möglich ist, durch den Einfluss des Lichtes selbst die Thiere nach Belieben positiv und negativ heliotropisch za machen. Die Larven von Balanus perforatus und die Larve gewisser Würmer und gewiss noch eine grosse Zahl anderer Thiere wurden, wenn sie längere Zeit im Dunkeln waren, positiv heliotropisch. Bringt man sie in Licht von genügender Intensität, so werden sie hier nach einiger Zeit negativ heliotropisch und zwar um so rascher, je intensiver das Licht ist.

Wir stossen also bei der Analyse der bewegungsbestimmenden Ursachen für die „freiwilligen“ Bewegungen eines Thieres nicht immer auf sehr einfache Verhältnisse; aber so komplizirt sie auch sein mögen: die „freiwilligen" Bewegungen der Thiere werden, wie unsere Erfahrung lehrt, doch immer nur dureh solche Umstände eindeutig bestimmt, welche auch in der unbelebten Natur die Bewegungen der Körper bestimmen.

5. Gewiss würden viele derjenigen Autoren, welche sich meinen Ausfübrungen widersetzen, protestiren, wenn man ron ihnen behaupten wollte, sie hielten „den Willen“ für etwas, das nicht auf physikalischem oder chemischem Wege seine Erklärung finden würde. Aber zeigt man das physikalische Agens, welches dem thierischen Körper seine Orientirung oder die Richtung seiner Bewegung eindeutig vorschreibt, welche man vorher als durch ,den 
Willen" bestimmt glaubte, so ist man enttäuscht, unzufrieden. Man zweifelte nicht an der physikalischen Lösung der Sache, aber man erwartete doch etwas „Höheres", etwas mehr der Ganglienzellenmystik Aehnliches. Gewiss ist mit dem Nachweis, dass die Richtung des Lichtstrahls den Hämatokokkusschwärmern oder den Balanidennauplien die Richtung der Progressivbewegung vorschreibt, unsere Kenntniss dieses Vorganges noch nicht ersehöpft; so wenig wie die physikalische Einsicht in das Zustandekommen der chemischen Lichtwirkungen heute erschöpft ist. Und doch wird deshalb Niemand sagen, dass ein Instinkt bei den erwähnten physikalischen Erscheinungen der die Umwandlung bestimmende Umstand sei.

6. Wie die vorangegangene Generation von Physiologen es als einen hemmenden Umstand empfand, dass man nicht die Ursachen der Erscheinungen an lebenden Organismen feststellte, wie das in der unbelebten Natur Sitte war, sondern, dass man sich mit der Phrase, die Lebenskraft sei die Ursache, zufrieden gab; so ist es für uns ebenso hemmend, dass auf dem allerdings kleineren Gebiet der sogenannten psychischen Lebenserscheinungen der Einfluss jener scholastischen Denkweise sich bis heute erhalten hat. Die Hemmung besteht darin, dass mit der Phrase, der Instinkt oder der freie Wille bestimme die Bewegung, die wirkliche Aufgabe der naturwissenschaftlichen Forschung auf diesem Gebiete nicht erkannt oder verdeckt wird: nämlich die bewegungsbestimmenden Umstände in jedem Falle einer ,freiwilligen“ Bewegung eines Thieres festzustellen. Es war Zweck dieser Zeilen darauf hinzuweisen, dass wir ebenso unbekümmert um den „Instinkt" und den "Willen", wie um die "Lebenskraft" diese Aufgabe zu erfüllen suchen müssen. 
Weitere Untersuchungen über den Heliotropismus der Thiere etc. 415

\section{Erklärung der Abbildungen anf Tafel IX und im Text.}

Fig. 1. Dauernde heliotropische Krümmungen bei Spirographis Spallanzanii. Die Thiere waren ursprünglich horizontal auf den Boden des Aquariums gelegt, der Mehrzahl nach mit dem. Kopf zur Zimmerseite e $f g h$ des Aquariums. Die Thiere krümmen sich so lange, bis ihr Kopf zur Lichtseite $c d i k$ des Aquariums gewendet ist, und die Axe des Kiemenblattes in der Richtung der Strahlen des Himmelslichtes steht. In verkleinertem Maassstab.

Fig. 2. Dieselben Thiere wie vorhin, nachdem das Aquarium um $180^{\circ}$ gedreht ist und die Lichtstrahlen mehr horizontal einfallen. Die Thiere haben den Kopf wieder der Lichtquelle zugewendet, wobei die bis dahin bestehenden heliotropischen Krümmungen wieder rückgängig gemacht wurden. Entsprechend dem mehr horizontalen Einfall der Lichtstrahlen ist die Orientirung der Axe des Kiemenblattes diesmal bei den Thieren mehr horizontal. In verkleinertem Maassstab.

Fig. 3a im Text. Schema der Orientirungsänderung der Spirographis beim Uebergang aus der Lage in Fig. 1 in diejenige von Fig. 2.

Fig. 3b. im Text. Orientirung der Spirographis gegen die Lichtquelle, wenn die heliotropische Krümmung durch die Glaswand $a b$ beeinträchtigt wird. Der Pfeil zeigt die Richtung der Lichtstrahlen an. Die Spirographis, in der Orientirung $M N$ ins Aquarium gebracht, nimmt die heliotropische Krümmung $M N_{1}$ an und setzt die Krümmungeu weiter fort, bis die Kiemen die Glaswand berühren; sie krümmt dann die Kiemen von der Glaswand weg (was in der Zeichnung weggelassen ist) und bleibt zuletzt dauernd in der Lage $\mathrm{M} \mathrm{N}_{2}$.

Fig. 4. Röhre von Spirographis Spallanzanii tnit dem neugebildeten Stücke a c $d e$ (natürliche Grösse). Vgl. Text S. 401.

Fig. 5. Heliotropische (und gotropische?) Krümmung der Röhre von Serpula uncinata durch Wachsthum. Zu Beginn des Versuches waren nur Röhrenelemente in der Richtung des Pfeiles $a b$ vorhanden. Der Block (der so gross war, dass nur ein kleines Stück desselben gezeichnet werden konnte) wurde so ins Aquariam gelegt, dass nunmehr das Licht in der Richtung des Pfeiles $c d$ die Röhren traf. Bei denjenigen Röhren nun, welche zu wachsen fortfuhren, krümmte sich die neu hinzuwachsende Spitze ausnahmslos in die Richtung der Lichtstrahlen (und der Schwerkraft) nach oben. Der freie Rand des Blocks war nach 6 Wochen mit solchen aufwärts gekrümmten Röhren übersäet. Natürliche Grösse. 
416 J.: Loeb: Weitere Untersuchungen über den Heliotropismus etc.

Fig. 6,7 u. 8 . Heliotropische Erscheinungen an wachsenden Elementen von Sertularia (polyzonias?). Die Stämme waren nahe der Wurzel bei $b$ abgeschnitten und mit der Spitze $a$ verkehrt in den Sand gesteckt. Das Stück $a$ e steckte im Sande.

Aus dem nach oben gerichteten Ende $b$ wuchsen sowohl Wurzeln $\left(W_{1}\right)$ wie Sprosse $S$; beide wuchsen in der durch Pfeile bezeichntien Richtung der Lichtstrahlen; die polypentragenden Sprosse aber zur Lichtquelle, die Wurzeln in der entgegengesetzten Richtung.

In Fig. 6 entstehen am alten Stamm Wurzeln $W_{2}$, welche ebenfalls negativ heliotropisch sind.

In Fig. 8 entsteht der Spross auf der der Lichtquelle zugekehrten oberen Seite der Wurzel.

Maassstab: 2 : 1 der natïrlichen Grösse. 


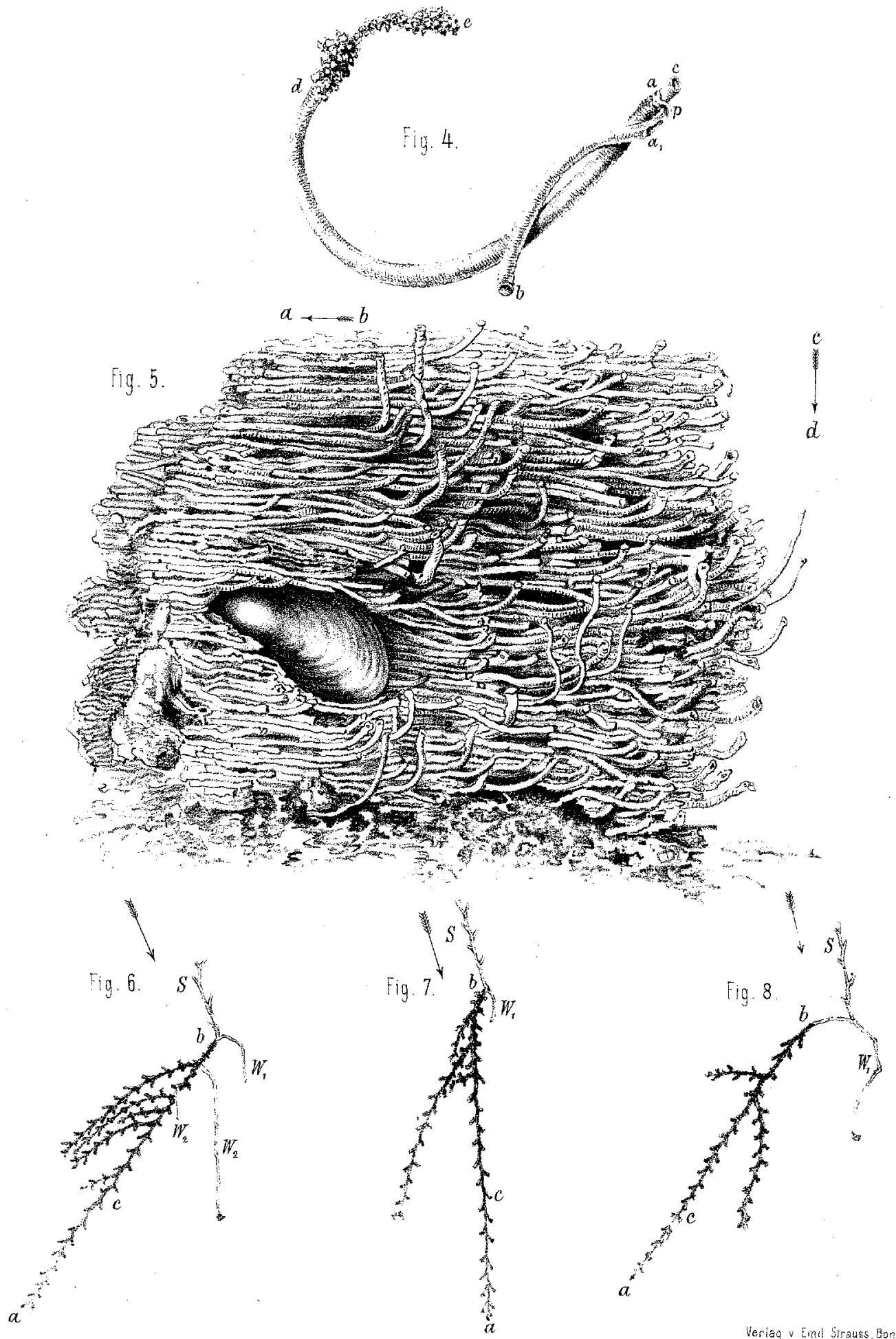

